\title{
Identification of the Electric Spark Electromagnetic Waveform Based on SVM
}

\author{
Tongtong $\mathrm{Li}^{1}{ }^{1,}$, Ziyuan Tong ${ }^{2}$, Shoufeng Tang ${ }^{1}$, Xia Qin ${ }^{1}$, Mingming Tong ${ }^{1}$ and Zhaoliang $\mathrm{Xu}^{3}$ \\ ${ }^{1}$ China University of Mining and Technology, China \\ ${ }^{2}$ School of Electrical Engineering and Telecommunications, The University of New South Wales, Australia \\ ${ }^{3}$ Xuzhou Hanlin Technology Co., Ltd., China \\ ${ }^{*}$ Corresponding author
}

\begin{abstract}
Electromagnetic wave of electrical spark is a potential cause to eletrical equipment failure. This research focused on identificating and comparative analyzing the different types of electromagnetic waveform generated by eletrical equipment failure based on SVM. After analyzing and extracting the features the electromagnetic waveform, a model was built to identificate the type of the elctromagnetic waveform. The collected standard electromagnetic waveforms were used as the imput of the train model and the model accuracy was improved by adjusting training parameters afer analyzing the results, When inputting an unknown type of electromagnetic waveform, SVM may predict the output of the network according to the recognition rule. Then the types of electromagnetic waveforms were identificated by using adjusted models. The result shows that the electromagnetic waveform can be effectively and feasibly identificated based on SVM, which provides a theoretical support on prediction method of gas explosion caused by electrical sparks.
\end{abstract}

Keywords-SVM; features of electromagnetic waveform; waveform identification

\section{INTRODUCTION}

Under the influence of the power frequency electric field ${ }^{[1]}$, the electric spark is a phenomenon of breakdown discharge when strong electric field ionizes the gas in the contact gap because of a momentary strong electric field or quick close and disconnection, together with a series of light, heat and other phenomena ${ }^{[2]}$, which resultes the resistance cannot continue to maintain the insulation property. Electric spark exhibits a wide frequency band distribution over the entire frequency band of electromagnetic waves shown in the recent studies ${ }^{[3]}$.

\section{THE EXTRACTION OF ELECTROMAGNETIC}

CHARACTERISTICS AND THE MODEL ESTABLISHMENT OF THE ELECTRIC SPARK WAVEFORMS

From the mechanism of the generation of electrical sparks, compared with the mechanism of static electricity and lightning lightning ${ }^{[4]}$, no matter what kind of spark discharge is used, there will be transient, high frequency, short rise time and short duration electromagnetic pulses in spark discharge. The electric spark is not generated by the static electric field generated by the initial accumulated electrostatic charge, but the electromagnetic pulse generated by the discharge is the same ${ }^{[5]}$. The electric spark pulse electromagnetic field is the electromagnetic field generated by the discharge transient current.

\section{A. Electromagnetic Characteristics Extraction of the Electric Spark}

First, confirm that you have the correct template for your paper size. This template has been tailored for output on the US-letter paper size. If you are using A4-sized paper, please close this file and download the file for "MSW A4 format". When the distance between the contact closure electrodes gradually decreases or a strong electric field occurs ${ }^{[6]}$, the current density gradually increases and produces high temperature ionization, then contact and electrode gas will be punctured. The moment of touch contaction or electric field reaching maximum leads to the discharge current reaches a peak, and then current decreases with exponential to zero ${ }^{[7]}$. So, the relationship between the voltage across the capacitor and the breakdown voltage can be described as follow:

$$
u_{C}=U_{C} e^{-\alpha}\left(\cos \omega t+\frac{\delta}{\omega} \sin \omega t\right)+\frac{u_{o}^{o} e^{-\alpha}}{t-\frac{T_{R C}}{T_{L R}}} \cdot \frac{4}{\pi} \sum_{n=1}^{\infty} \frac{\sin (2 n-1) \omega t}{2 n-1}
$$

The electric current passed through electric spark can be described as follow:

$$
I=C_{o} u_{C}^{\prime}=\frac{U_{C} e^{-\delta t}}{L \omega} \sin \omega t-\frac{C u_{o}^{o} e^{-\delta t}}{T_{L R}-T_{R C}} \cdot \frac{4}{\pi} \sum_{n=1}^{\infty} \frac{\sin (2 n-1) \omega t}{2 n-1}
$$

$$
\text { Where } T_{L R}=\frac{2 L}{R}, \omega=\sqrt{T_{C R}^{-2}-T_{L R}^{-2}}=\frac{\sqrt{\frac{T_{L R}}{T_{R C}}-1}}{T_{L R}}, \delta=\frac{R}{2 L}=\frac{1}{T_{L R}}
$$

The parameters of the electrical spark obtained by above formulas mainly include discharge voltage, discharge current, frequency, amplitude, energy, wavelength and rise time. Different types of discharge sparks have great differences in the four parameters ${ }^{[8]}$, namely amplitude, frequency, rise time, and discharge current. Therefore, the amplitude, frequency, rise time, discharge current, and discharge voltage are selected as the parameters of the electromagnetic wave, which are the object of the subsequent analysis. 


\section{B. Establishment of Electric Spark Magnetic Field Model Based on SVM}

Using SVM to identify electrical spark electromagnetic waves, we first select the parameters obtained by the wavelet transform of electrical spark electromagnetic wave of known discharge type to form a training sample set, which was used as the input of the SVM training module. Then use the training sample data to train the SVM to get the support vector and the corresponding parameters, and thus establish the SVM model. If the result accuracy of model meets the expected requirements ${ }^{[9]}$, it is saved for use in the identification process of discharge spark electromagnetic waves. If the result accuracy of the model does not meet the expected requirements, the training parameters are readjusted and training is performed until the accuracy of the training error reaches the expected. Finally, the parameter data of the discharge spark electromagnetic wave to be tested is input into the established SVM model to obtain the spark spark type recognition result.

The flow chart of the entire training and testing process is shown in Figure I.

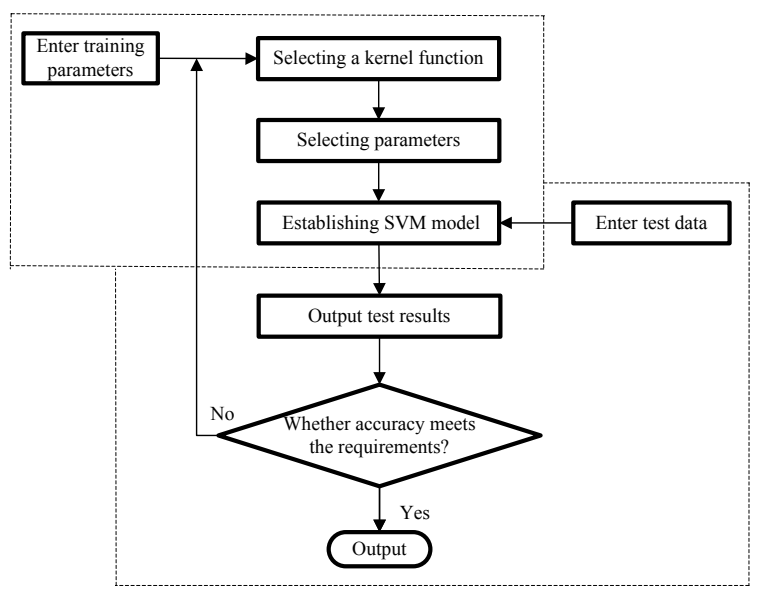

FIGURE I. SVM TRAINING AND TESTING FLOW

Support vector machine (SVM) model sample selection of three discharge types: low-voltage discharge sparks, highvoltage discharge sparks and glow discharge sparks. After wavelet analysis of electromagnetic wave data, amplitude, frequency, rise time, discharge Voltage and discharge current were selected. Due to the different dimensions of the five parameters, before the model training, the sample data need to be normalized to eliminate the influence of different dimensions and differences in the size of the different indicators on the recognition results ${ }^{[10]}$. Use the svdatanorm command in MATLAB to normalize the sample data.

The support vector machine uses the binary tree method to identify the type of discharge sparks ${ }^{[11]}$. In fact, the discriminant function $\mathrm{fl}$ is calculated from the root node, and the discharge spark type 1 and other types of discharge sparks are distinguished based on the value of $\mathrm{fl}$. If $\mathrm{fl}=-1$, then the input spark is type 1 . if $f 1=1$, then $f 2$ is calculated, and so on. Until the state combination of the discriminant functions $\mathrm{fl}, \mathrm{f} 2$, f3. Complete the identification of discharge spark electromagnetic waves, as shown in Table I.
TABLE I. SVM DECISION RULES TO RECOGNIZE MULTIHEADSTREAMS

\begin{tabular}{ccc}
\hline Types of Electric Spark & $f_{1}$ & $f_{2}$ \\
\hline 1 High-voltage discharge spark & -1 & +1 \\
2 Low-voltage discharge spark & +1 & -1 \\
3 Glow discharge spark & +1 & +1 \\
\hline
\end{tabular}

Finally, MATLAB was used to program in SVM toolbox ${ }^{[12]}$, support vector machine and training function svc were used to design for SVM classifier design and train samples.

\section{The Processing AND ANALysis of DATA}

Using the SVM model that has been trained, the actual output of the spark discharge spark sample is used to complete the identification work and compared with the actual situation. As shown in Table II, HV-discharge spark represents Highvoltage discharge spark, LV-discharge spark represents Lowvoltage discharge spark, and G-discharge spark represents Glow discharge spark. the SVM model is used to identify 25 samples, the number of correct data is 23 , the correct rate was $92 \%$. It can be seen that using the binary tree SVM model to identify the type of discharge sparks has a higher correct rate.

TABLE II. RECOGNITION RESULTS OF SVM

\begin{tabular}{|c|c|c|c|c|}
\hline No. & $f_{1}$ & $f_{2}$ & $\begin{array}{c}\text { Recognition result } \\
\text { of SVM }\end{array}$ & The actual situation \\
\hline 1 & -1 & +1 & HV-discharge spark & HV-discharge spark \\
\hline 2 & -1 & +1 & HV-discharge spark & HV-discharge spark \\
\hline 3 & -1 & +1 & HV-discharge spark & HV-discharge spark \\
\hline 4 & -1 & +1 & HV-discharge spark & HV-discharge spark \\
\hline 5 & -1 & +1 & HV-discharge spark & HV-discharge spark \\
\hline 6 & -1 & +1 & HV-discharge spark & HV-discharge spark \\
\hline 7 & -1 & +1 & HV-discharge spark & HV-discharge spark \\
\hline 8 & -1 & +1 & HV-discharge spark & HV-discharge spark \\
\hline 9 & +1 & -1 & LV-discharge spark & LV-discharge spark \\
\hline 10 & +1 & -1 & LV-discharge spark & LV-discharge spark \\
\hline 11 & +1 & -1 & LV-discharge spark & LV-discharge spark \\
\hline 12 & +1 & -1 & LV-discharge spark & LV-discharge spark \\
\hline 13 & +1 & -1 & LV-discharge spark & LV-discharge spark \\
\hline 14 & +1 & +1 & G-discharge spark* & LV-discharge spark \\
\hline 15 & +1 & -1 & LV-discharge spark & LV-discharge spark \\
\hline 16 & +1 & -1 & LV-discharge spark & LV-discharge spark \\
\hline 17 & +1 & -1 & LV-discharge spark & LV-discharge spark \\
\hline 18 & +1 & +1 & G-discharge spark & G-discharge spark \\
\hline 19 & +1 & +1 & G-discharge spark & G-discharge spark \\
\hline 20 & +1 & +1 & G-discharge spark & G-discharge spark \\
\hline 21 & -1 & -1 & HV-discharge spark* & G-discharge spark \\
\hline 22 & +1 & +1 & G-discharge spark & G-discharge spark \\
\hline 23 & +1 & +1 & G-discharge spark & G-discharge spark \\
\hline 24 & +1 & +1 & G-discharge spark & G-discharge spark \\
\hline 25 & +1 & +1 & G-discharge spark & G-discharge spark \\
\hline
\end{tabular}

\section{CONCLUSION}

Based on the SVM, the network model of the discharge spark electromagnetic wave was established and trained. In the case of the insufficient number of spark spark samples, it can also play a good classification and recognition role. Through 
the trained network, 25 groups of spark samples are identified, the recognition rate is high and the training algorithm time is very short. It solves practical problems such as small sample, nonlinear and high-dimensional pattern recognition, and determines the SVM discharge. The effectiveness and feasibility of spark electromagnetic wave recognition make the recognition model have good generalization performance.

\section{ACKNOWLEDGMENT}

This project is supported by the National key research and development program (Grant No.2016YFC0801800)

\section{REFERENCES}

[1] N. Fujimoto and S. A. Boggs. Characteristics of risetime transients incident on externally connected GIS disconnector induced shortpower system components[J]. Transaction on Power Delivery, 1988, 3(3):961970.

[2] Delyon B,Juditsky A. Accuracy Analysis for Wavelet Approximations[C]. IEEE Transactions on Neural Networks, 1995,6(2): 332-348.

[3] Zoran Radojević;;Vladimir TerzijaIntelligent two-port numerical algorithm for transmission lines disturbance records analysis[J] Electrical Engineering 2013-5.

[4] SolarBOS; SolarBOS Passes Arc Fault Test in Livermore, CA[J]Anonymous Energy Weekly News 2011.

[5] Temple, David;Joye, Ken Consulting.A look at arc-resistant switchgear[J] Specifying Engineer 2009-6.

[6] Sham Sunder Srinivas Yellamraju;;Sanjay V. Kulkarni .Performance and modelling of $70 \mathrm{kVdc}$ power supply with solid-state crowbar[J]Fusion Engineering and Design 2013.

[7] Walsh, Peter R Consulting .Calculating Arc Flash Hazard Levels[J] Specifying Engineer 2008-6.

[8] C.E.R.Bruce and R.H.Golde, The Lightning Discharge[J]. J. Inst. Elect. Engrs. 1941, (88):487505.

[9] Elio Fonseca Barbosa, Jose Osvaldo Saldanha Paulino, Glassio Costa de Miranda. Measured and Modeled Horizontal Electric Field From Rocket-Triggered Lightning[C]. IEEE Trans. Electromagn. Compat,2008, 4(50):913-920.

[10] Pan Q. Two Denoising Methods by Wavelet Transform[C]. Signal Processing, 1999, 47(3): 3401-3405.

[11] Gedney S D. An anisotropic perfectly matched layer absorbing media for the truncation of FDTD lattices[C]. pp1630-1639. trans. Antennas Propagat., Dec. 1996, AP-44(12).

[12] Chui.C.K.Wavelet. A tutorial theory and applieations [C]. NewYork: A Cademic Fress, 1992:1-453. 